\title{
What do patients think of patient-maintained sedation?
}

Patient-maintained sedation for oral surgery using a target-controlled infusion of propofol - a pilot study

\author{
A. Leitch, N. Sutcliffe and G. N.C. Kenny Br Dent J 2003; 194: 43-45
}

Objective

To assess the safety and efficacy of a new patient-maintained propofol system for conscious sedation in dentistry.

Design

Prospective clinical trial

Setting

Department of Sedation, Glasgow Dental Hospital and School. 2001

Subjects and methods

Patients scheduled for oral surgery with conscious sedation. Exclusions included ASA IV -V, inability to use the handset, opioid use and severe respiratory disease.

\section{Interventions}

Patients were given intravenous propofol to a level of $1.0 \mu \mathrm{g} / \mathrm{ml}$ (reducing from $1.5 \mu \mathrm{g} / \mathrm{ml}$ ) using a target controlled infusion system, they then controlled their sedation level by double-clicking a handset which on each activation increased the propofol concentration by $0.2 \mu \mathrm{g} / \mathrm{ml}$.

Main outcome measures

Oxygen saturation, patient satisfaction, and surgeon satisfaction.

\section{Results}

Twenty patients were recruited, 16 female and four male. Nineteen patients completed sedation and treatment successfully. Mean lowest oxygen saturation was 94\%. No patients were oversedated. All patients successfully used the system to maintain a level of sedation adequate for their comfort. Patient and surgeon satisfaction were consistently high.

\section{Conclusions}

Initial experience with this novel system has confirmed safety, patient satisfaction and surgeon satisfaction.

\section{IN BRIEF}

- Intravenous sedation with Midazolam is well established but is not suitable for all clinical situations.

- Patient-maintained sedation with propofol may offer an alternative to midazolam.

- The system is safe and reproducible.

- Patient and surgeon satisfaction are high.

- Recovery is fast and uneventful.

\section{COMMENT}

Target-controlled infusion ( $\mathrm{TCl}$ ) with propofol is gaining wide popularity within anaesthesia. The system uses a pharmacokinetic model to achieve and maintain a target blood level of propofol via the use of a computer controlled infusion pump. This clinical trial has further developed the technique by incorporating a handset to allow the patient to titrate the propofol to a level of sedation that suits their individual needs - so called patient maintained sedation (PMS). The aim is to combine the benefits of TCl and PMS.

Since its introduction as a general anaesthetic induction agent, propofol has attracted interest as a sedative agent. At present midazolam is the principle drug used for intravenous dental sedation in the UK and although an excellent drug, it does have the disadvantage of prolonged recovery. With propofol, induction and recovery is so rapid the inter-operative level and length of sedation can be readily adjusted. Upon completion recovery is very rapid with minimal CNS effects when compared with other sedative agents. However, propofol is not currently licensed as a dental intravenous sedative agent and can only be used by anaesthetists and those specifically trained in intensive care.

The 20 patients (age range 12 to 58 years old) in this study were non-selectively recruited and represent the normal range of patients expected in an outpatient oral surgery department. Each patient received intravenous propofol to a target blood level but they were then allowed to control their sedation level via the use of a handset. Patients were monitored throughout the procedure. Primary outcome measures were physiological signs (oxygen saturation, heart rate and blood pressure), patient satisfaction and operator satisfaction.

The results demonstrated a high patient satisfaction with the technique despite the apparent lack of amnesia. Operator satisfaction also rated highly in all but one case (a 12-year-old child). Physiological parameters were stable with only one case of transient desaturation, an event that would not be unusual with midazolam sedation. One patient suffered a series of epileptic fits requiring hospital admission. The authors and the data sheet warn that special care is needed in treating epileptics with propofol.

Despite the modest number of patients and the wide range of ages, this paper successfully describes a new technique for the administration of propofol intravenous sedation. Further research work, in the form of a randomised controlled trial, is required to confirm the efficacy this technique.

Dr John Lyne, Sedation Practitioner, Newcastle Dental Hospital and School 